\title{
Metal Artifact Reduction from Computed Tomography (CT) Images using Directional Restoration Filter
}

\author{
Mithun Kumar PK, Mohammad Motiur Rahman \\ Department of Computer Science \& Engineering, Mawlana Bhashani Science and Technology University, Tangail- \\ 1902, Dhaka, Bangladesh \\ Email:*mithun24bd@gmail.com,mm73rahman@gmail.com
}

\begin{abstract}
Computed tomography angiography (CTA) is a stabilized tool for vessel imaging in the medical image processing field. High-intense structures in the contrast image can seriously hamper luminal visualization. Metal artifacts are an extensive problem in computed tomography (CT) images. We proposed directional restoration filtering process with Fuzzy logic in order to reduce metal artifact from CT images. We create two sets by iteration process and these sets will be sorted in ascending order. After sorting we take two elements from two data sets and the tracking both elements will be selected from the second position of those sorting arrays. Intersection Fuzzy logic will be executed between two selected elements and Gaussian convolution operation will be performed in the entire images because of enhancement the artifact affected CT images. In this paper, we investigated a fully automated intensity-based filter and it depends on the gray level variation rating. This results in a better visualization of the vessel lumen, also of the smaller vessels, allowing a faster and more accurate inspection of the whole vascular structures.
\end{abstract}

Index Terms - Artifact reduction, Filter, Stent wire, Computed Tomography, Restoration, Gaussian convolution

\section{INTRODUCTION}

Now a day's medical image processing is a most important task in diagnosis of diseases. Computed tomography (CT) [1] is highly noninvasive compared to other medical imaging modalities (e.g. Ultrasound imaging, Magnetic resonance). Unfortunately, CT image is corrupted by metal artifact when stent wire is used in cardiac functionalities. We are interested in metal artifact reduction in computed tomography (CT) reconstruction. The application subcontext is that of vascular imaging in the presence of a metal stent. Metal artifact reduction is one of the most important preprocessing tasks in the computed tomography processing field and it is a very complex and critical preprocessing step for feature extraction, segmentation, classification, registration from medical CT images.

Various artifact reduction techniques have been proposed, namely, statistical reconstruction algorithms [2] are more robust to the data inconsistencies that cause these artifacts. Most modern scanners implement various data preprocessing techniques to reduce these artifacts, many of which can amplify data noise [3]. [4] Used statistical reconstruction procedure on a sinogram that had been preprocessed by a metal artifact method. Moreover, metal artifact reduction by sinogram subtraction and simplistic interpolation may induce other artifacts in reconstructed images [5]. Many image denoising methods are proposed basis on wavelet, local statistics, contourlet transform, non-linear adaptive thresholding [6-34]. Extra-Energy Reduction (EER) [20] function is formulated based on local degree of unsmooth pixels in order to imperative results as well as create stable and equilibrium state in the entire images. Laplacian pyramid-base nonlinear diffusion (LPND) method [35] used for speckle reduction and edge preservation. The automatic determination of the gradient threshold used in the LPND. LPND method used $M A D$ (Median Absolute Deviation) operator [36] for determination of the gradient threshold. An edge sensitive diffusion method [37] [i.e. speckle reducing anisotropic diffusion (SRAD)] is the speckle reducing anisotropic nonlinear diffusion filtering approach to spatial adaptive filtering as well as it demonstrates effective results of denoising in homogeneous regions in an image.

Our proposed method is based on the intersection fuzzy logic and iterative reconstruction in getting data set from the directional image data structure. The method does not require a lot of memory to run and does not require a lot of time to converge final consequences. The introducing method is more convenient for real life application and provides large amount of clinical benefits and faster algorithm for reducing metal artifact from CT images as well. The prime purpose of our investigated method is to reduce metal artifact from CT images.

This paper is structured as follows. In Section 2, the theoretical background of the proposed method, including structural representation of the filtering technique, is presented. Section 3 describes evaluation criteria for checking performance with other state-of-theart algorithms (e.g. Perona-Malik (P-M) [34], SRAD [37], LPND [35] and EER [20]). Section 4 shows experimental visual results and graphical representation for performance testing. Section 5 offers concluding remarks. 


\section{PROPOSED METHOD}

We proposed direction oriented iterative reconstruction filtering process with Fuzzy logic for reducing metal artifact from CT images. Our investigated method based on a fully automated intensity variation while iterative method is used for estimating metal artifact pixel values from the experimental images. We create two sets by iteration process and these sets will be sorted in ascending order. After sorting we take two elements from two data sets at the target point. The tracking both elements will be selected from the second position of those sorting arrays. Then Fuzzy intersection logic will be executed between two selected elements and Gaussian convolution operation will be performed in the entire image. In figure 1 , we consider $A$ and $B$ direction oriented data sets which are generated from the original CT image $I(x, y)$, where image size is $X \times Y$.

Set $A$ is generated by following procedure:

$$
A=\boldsymbol{R}_{i=-(r / 2)}^{r / 2} I(x+i, y)
$$

And similarly set $B$ is generated by following procedure:

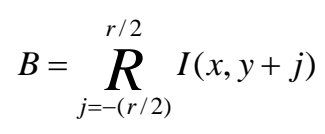

Where big- $\boldsymbol{R}$ is the iterative operator and it has been adopted and implemented in Real-Time Process Algebra (RTPA) [38] and $r$ is the total number of element of the data sets in each step of filter execution. Where $r=5$ and it is a positive odd integer number.

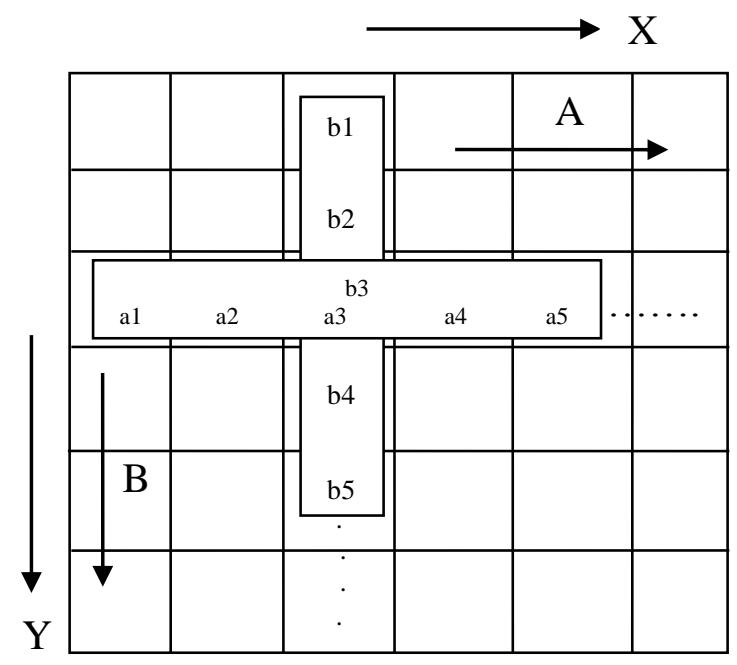

Fig. 1. structural representation of the filter mechanism

Now $A_{s}$ and $B_{s}$ are sorted data array in ascending order which are getting from $A$ and $B$ respectively. We track second position data element that are $A_{s}(2)$ and $B_{s}(2)$. We apply intersection Fuzzy logic between $A_{s}(2)$ and $B_{s}(2)$. If intersection Fuzzy set is $C$,

$$
\begin{gathered}
\mu_{C}(t)=\min \left(\mu_{A_{s}}(t), \mu_{B_{s}}(t)\right) \\
=\mu_{A_{s}}(t) \wedge \mu_{B_{s}}(t) \\
I^{n+1}(x, y)=I^{n}(x, y)=\mu_{C}(t)
\end{gathered}
$$

Where superscript $n$ is the number of iteration and $t=2$ is the target point in the selected data array, which is the most important task for getting significant desire result. We know metal artifact is mainly occurred in the high intensity gray level of the CT images, so we have to take appropriate decision about target point selection for super artifact reduction from $\mathrm{CT}$ images.

Let us consider a rectangular filtered image domain $\Omega:=[0, \mathrm{q} 1] \times[0, \mathrm{q} 2]$ with boundary $\Gamma:=\partial \Omega$ and let a gray-scale filtered image $I$ be represented by a real-valued mapping $I \in \mathrm{L}^{\infty}(\Omega)$. A widely-used way to smooth $I$ is by calculating the convolution

$$
\left(K_{\sigma} * I\right)(x):=\int_{\Omega} K_{\sigma}(x-y) I(y) d y
$$

Where $K_{\sigma}$ denotes the two-dimensional Gaussian of width (standard deviation) $\sigma>0$ :

$$
K_{\sigma}(x):=\frac{1}{2 \pi \sigma^{2}} \cdot \exp \left(-\frac{|x|^{2}}{2 \sigma^{2}}\right)
$$

Gaussian convolution mainly is used for smoothing an image and the standard deviation is the degree of smoothness in the image. If we consider large standard deviation then we need large kernel size for better result because large standard deviation provides higher degree of smoothness.

\section{EVALUATION CRITERIA}

We investigate the filter performance by applying two metrics Mean Square Error (MSE) [20] and Edge Preservation Factor (EPF) [20].The edge preservation ability of the filter is compared by Edge Preservation Factor and it is computed using (EPF):

$$
E P F=\frac{\sum(\Delta I-\overline{\Delta I})\left(\Delta I_{d}-\overline{\Delta I_{d}}\right)}{\sqrt{\sum(\Delta I-\overline{\Delta I})^{2}\left(\Delta I_{d}-\overline{\Delta I_{d}}\right)^{2}}}
$$

Where $\Delta I$ and $\Delta I_{d}$ are the high pass filtered versions of images $I$ and $I_{d}$, obtained with a $3 \times 3$ pixel standard approximation of the Laplacian operator. The larger value of EPF means more ability to preserve edges.

Mean Square Error (MSE):

$$
\mathrm{MSE}=\left[\frac{\sum_{x=0}^{M-1} \sum_{y=0}^{N-1}\left(I(x, y)-I_{d}(x, y)\right)^{2}}{M x N}\right]
$$


Where the image size is $M \times N$. $x$ means row, $y$ means column, $I$ means original image and $I_{d}$ means filtered image.

\section{EXPERIMENTAL RESULT}

The proposed method has been applied to 2-D axial CT image with have been corrupted by metal artifact. The computation is carried out on MATLAB 7.12.0.635(R2011a) in a Core i5 $2.50 \mathrm{GHz}$ and 4GB RAM laptop having a Windows 7 operating system. Here, we show the filtered images and try to depict the performance of filters about metal artifact reduction in Table 1-4 and Visual performance is also shown in figure 1-7 respectively.

Here, we represent our experimental result in subjective about artifact reduction in axial CT images. Filtered images are given below for making sense about filter performance:

From figure 2 and 3, we see that filtered images are more stable and clear rather than previous metal artifacts affected images and the huge amount of artifact is reduced from the original images as well as the investigated filter creates the highly smoothing and better visual quality in the same time as well. So, we can say that filter is more powerful and efficient for artifact reduction for making stabilized filtered images.

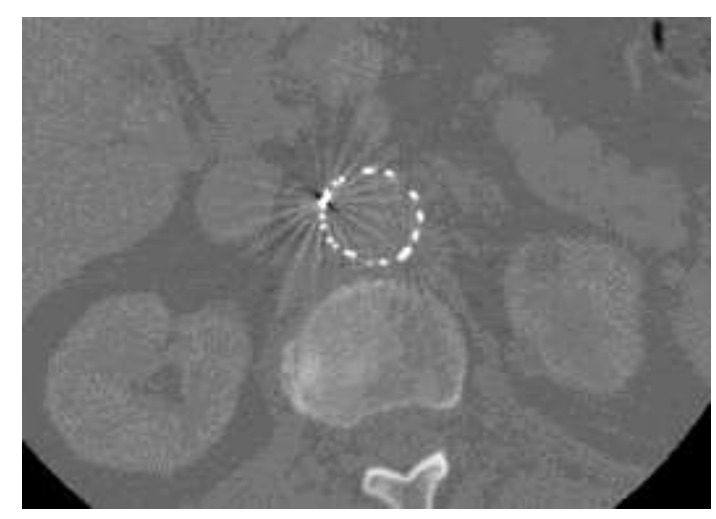

(a)

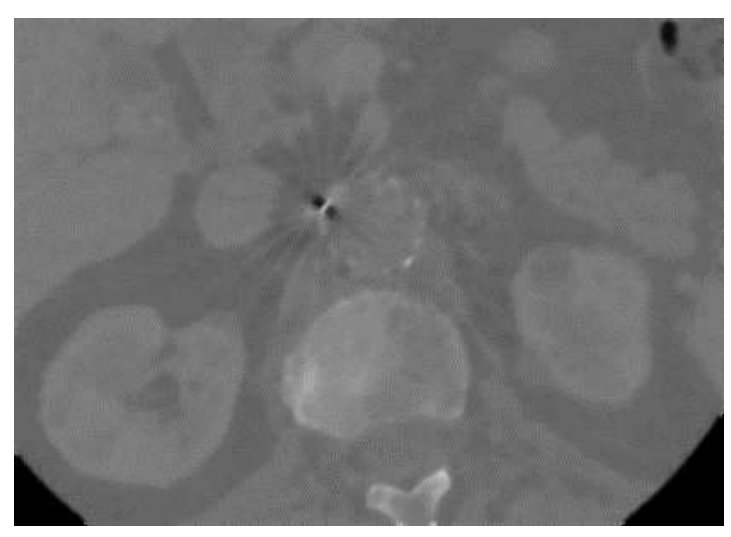

(b)

Fig. 2. 2-D axial CT image1 with artifacts present in fenestrated superior mesenteric stent, (a) before filtering image and (b) after filtering image

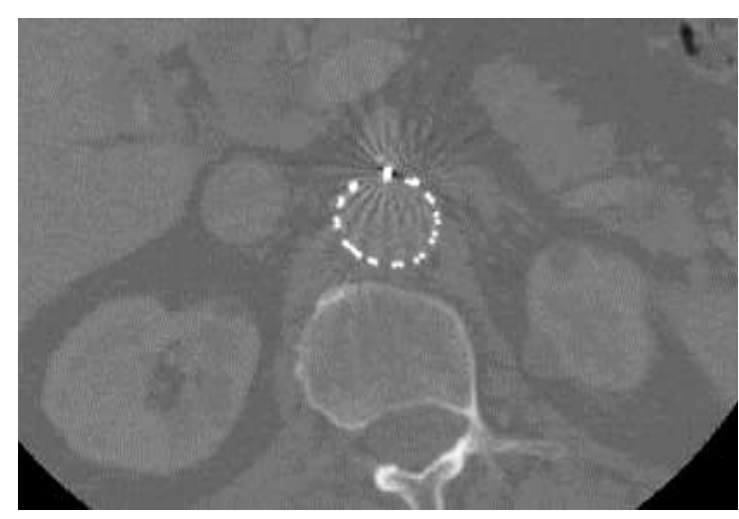

(a)

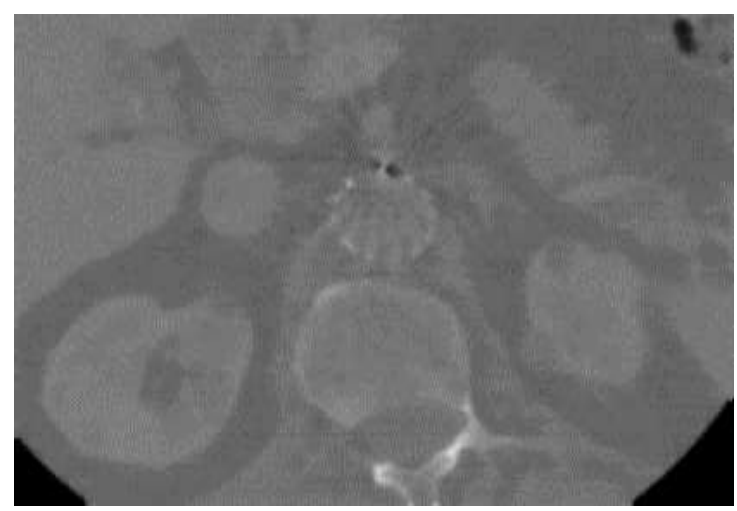

(b)

Fig. 3. 2-D axial CT image2 with artifacts present in fenestrated superior mesenteric stent, (a) before filtering image and (b) after filtering image

Graphical representation is the most important scientific parameter for judgments of the any types of imaging operation and the contrast measurement is another valuable approach for image quality judgment. If in the filtered images remain small amount of variation which reflects in the measurement curve then this filter suitability must be better for any kinds of imaging operations. Histogram of original images and filtered images are given below:

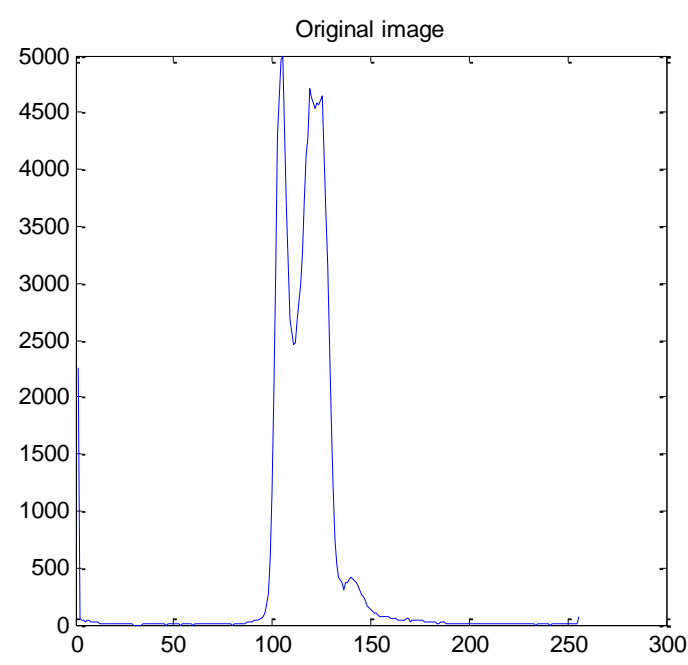

(a) 


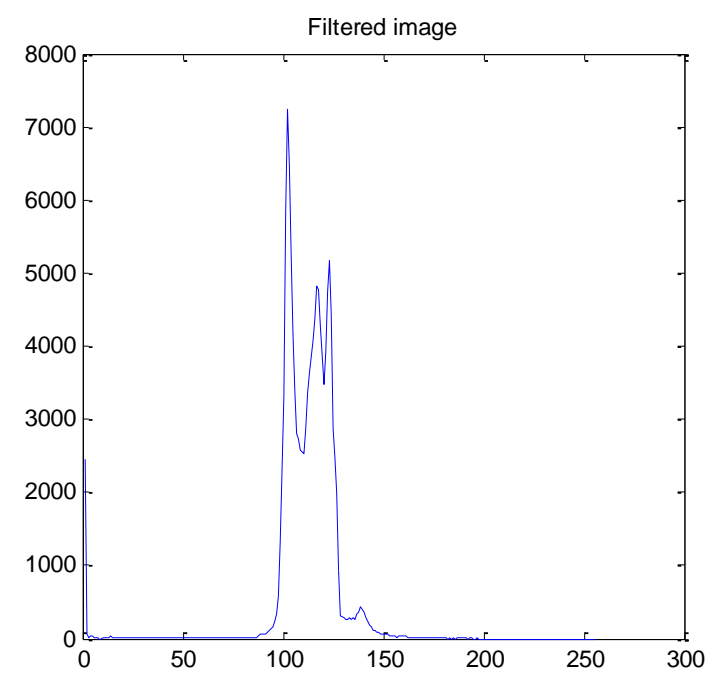

(b)

Fig. 4. 2-D axial CT image1, (a) Original image histogram and (b) Filtered image histogram

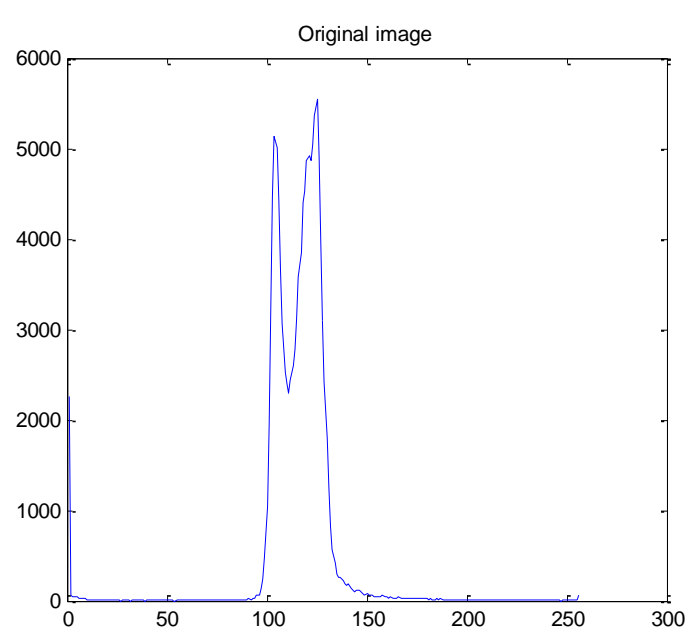

(a)

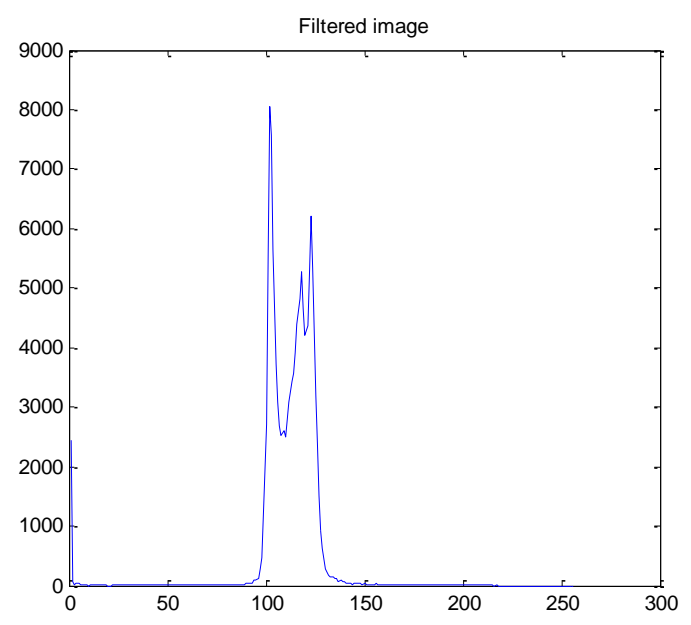

(b)

Fig. 5. 2-D axial CT image2, (a) Original image histogram and (b) Filtered image histogram
From figure 4 and 5, we see that filtered images are maintained structural view and enhance the contrast of the artifact corrupted images by reducing metal artifact from the axial CT images.

Statistical comparisons among some state-of-the-art algorithms are given below:

Table 1. Statistical performance of filters for 10 iterations

\begin{tabular}{|c|c|c|c|c|}
\hline \multirow{2}{*}{ Methods } & \multicolumn{2}{|c|}{ axial CT image1 } & \multicolumn{2}{c|}{ axial CT image2 } \\
\cline { 2 - 5 } & EPF & MSE & EPF & MSE \\
\hline Perona-Malik & 0.9767 & 33.1962 & 0.9777 & 32.7307 \\
\hline SRAD & 0.9658 & 39.5125 & 0.9665 & 38.5421 \\
\hline LPND & 0.8841 & 62.3937 & 0.8892 & 62.3974 \\
\hline EER & 0.9738 & 6.4990 & 0.9793 & 6.4801 \\
\hline Proposed & $\mathbf{0 . 9 7 9 6}$ & $\mathbf{0 . 0 7 5 7}$ & $\mathbf{0 . 9 8 2 8}$ & $\mathbf{0 . 0 7 4 7}$ \\
\hline
\end{tabular}

Table 2. Statistical performance of filters for 30 iterations

\begin{tabular}{|c|c|c|c|c|}
\hline \multirow{2}{*}{ Methods } & \multicolumn{2}{|c|}{ Stent wire image1 } & \multicolumn{2}{c|}{ Stent wire image2 } \\
\cline { 2 - 5 } & EPF & MSE & EPF & MSE \\
\hline Perona-Malik & 0.9383 & 93.0890 & 0.9580 & 92.4121 \\
\hline SRAD & 0.9257 & 41.1252 & 0.9274 & 41.2351 \\
\hline LPND & 0.8830 & 62.5916 & 0.8885 & 62.6194 \\
\hline EER & 0.9761 & 6.4981 & 0.9799 & 6.3806 \\
\hline Proposed & $\mathbf{0 . 9 7 9 6}$ & $\mathbf{0 . 0 7 5 7}$ & $\mathbf{0 . 9 8 2 8}$ & $\mathbf{0 . 0 7 4 7}$ \\
\hline
\end{tabular}

Table 3. Statistical performance of filters for 50 iterations

\begin{tabular}{|c|c|c|c|c|}
\hline \multirow{2}{*}{ Methods } & \multicolumn{2}{|c|}{ Stent wire image1 } & \multicolumn{2}{c|}{ Stent wire image2 } \\
\cline { 2 - 5 } & EPF & MSE & EPF & MSE \\
\hline Perona-Malik & 0.8712 & 115.6091 & 0.9123 & 116.2183 \\
\hline SRAD & 0.9058 & 43.9022 & 0.9064 & 44.6547 \\
\hline LPND & 0.8822 & 62.7793 & 0.8878 & 62.8186 \\
\hline EER & 0.9781 & 6.4901 & 0.9803 & 6.1302 \\
\hline Proposed & $\mathbf{0 . 9 7 9 6}$ & $\mathbf{0 . 0 7 5 7}$ & $\mathbf{0 . 9 8 2 8}$ & $\mathbf{0 . 0 7 4 7}$ \\
\hline
\end{tabular}

Table 4. Statistical performance of filters for 100 iterations

\begin{tabular}{|c|c|c|c|c|}
\hline \multirow{2}{*}{ Methods } & \multicolumn{2}{|c|}{ Stent wire image1 } & \multicolumn{2}{c|}{ Stent wire image2 } \\
\cline { 2 - 5 } & EPF & MSE & EPF & MSE \\
\hline Perona-Malik & 0.8652 & 138.9465 & 0.8871 & 141.5765 \\
\hline SRAD & 0.8740 & 45.7412 & 0.8714 & 47.9514 \\
\hline LPND & 0.8798 & 63.1864 & 0.8858 & 63.2603 \\
\hline EER & 0.9778 & 6.4851 & 0.9781 & 7.2805 \\
\hline Proposed & $\mathbf{0 . 9 7 9 6}$ & $\mathbf{0 . 0 7 5 7}$ & $\mathbf{0 . 9 8 2 8}$ & $\mathbf{0 . 0 7 4 7}$ \\
\hline
\end{tabular}

From Table 1, 2, $3 \&$ 4, We can observe that proposed filter is more efficient for metal artifact reduction from 
CT images than others existing well defined algorithms. The investigated method can reduce artifact significantly while edges, textures and point targets are smoothed as well as preserved without loss of any clinical information. Our filter alleviate artifact but do not make additional errors or noises in the filtered image which thing is mentionable with respect to others filter performance comparatively. In this experiment we did not use iteration for our filter but other filters are iterative.

Visual comparisons are given below with respect to our filter:
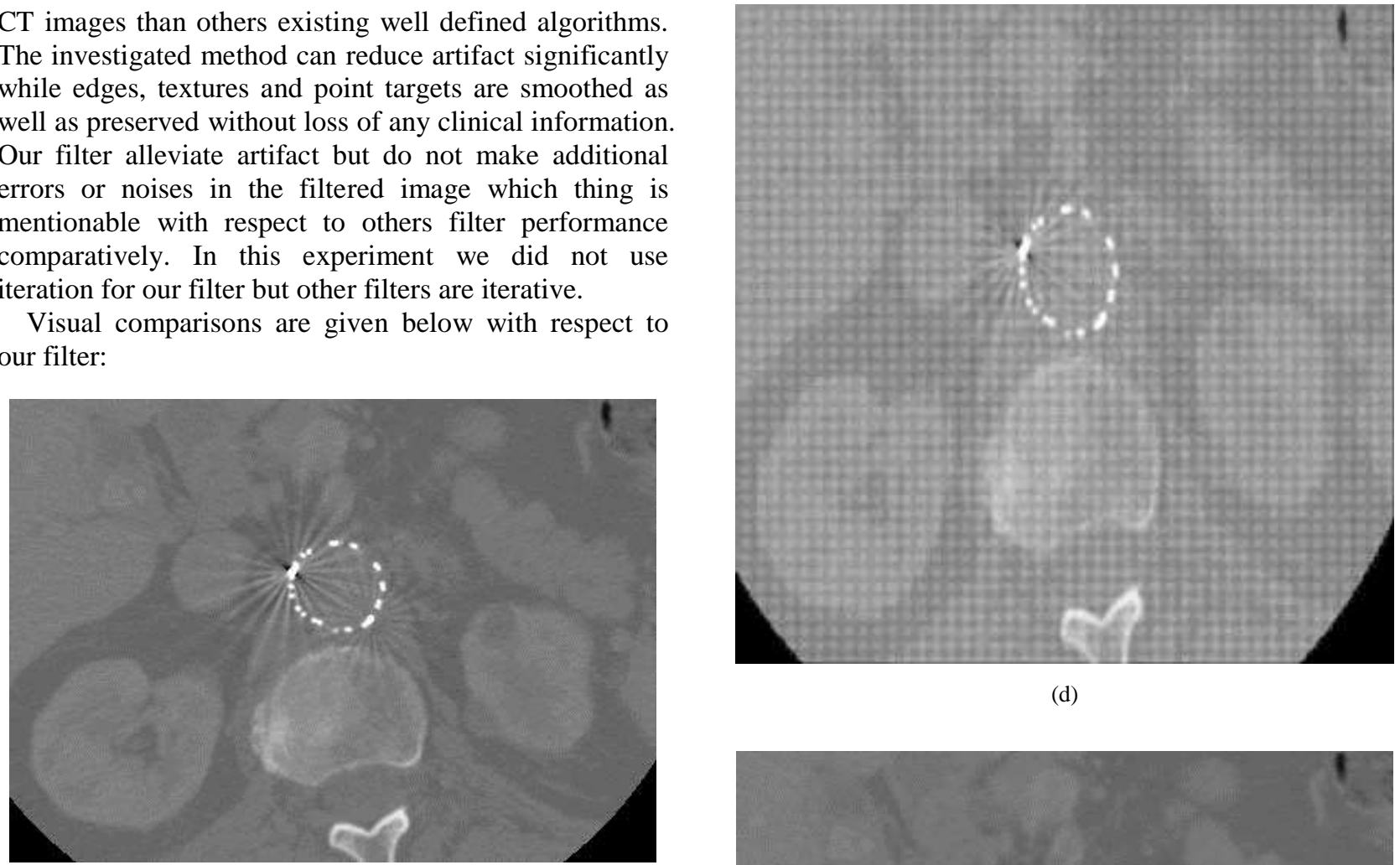

(d)

(a)

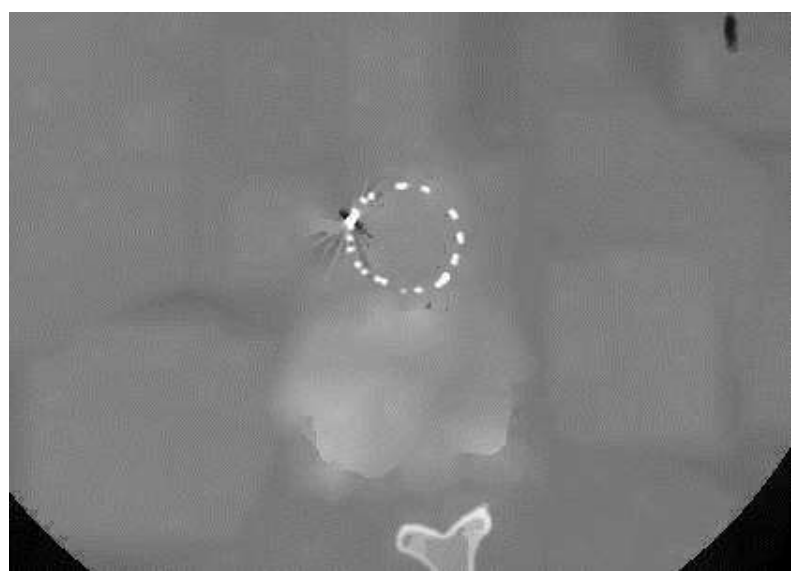

(b)
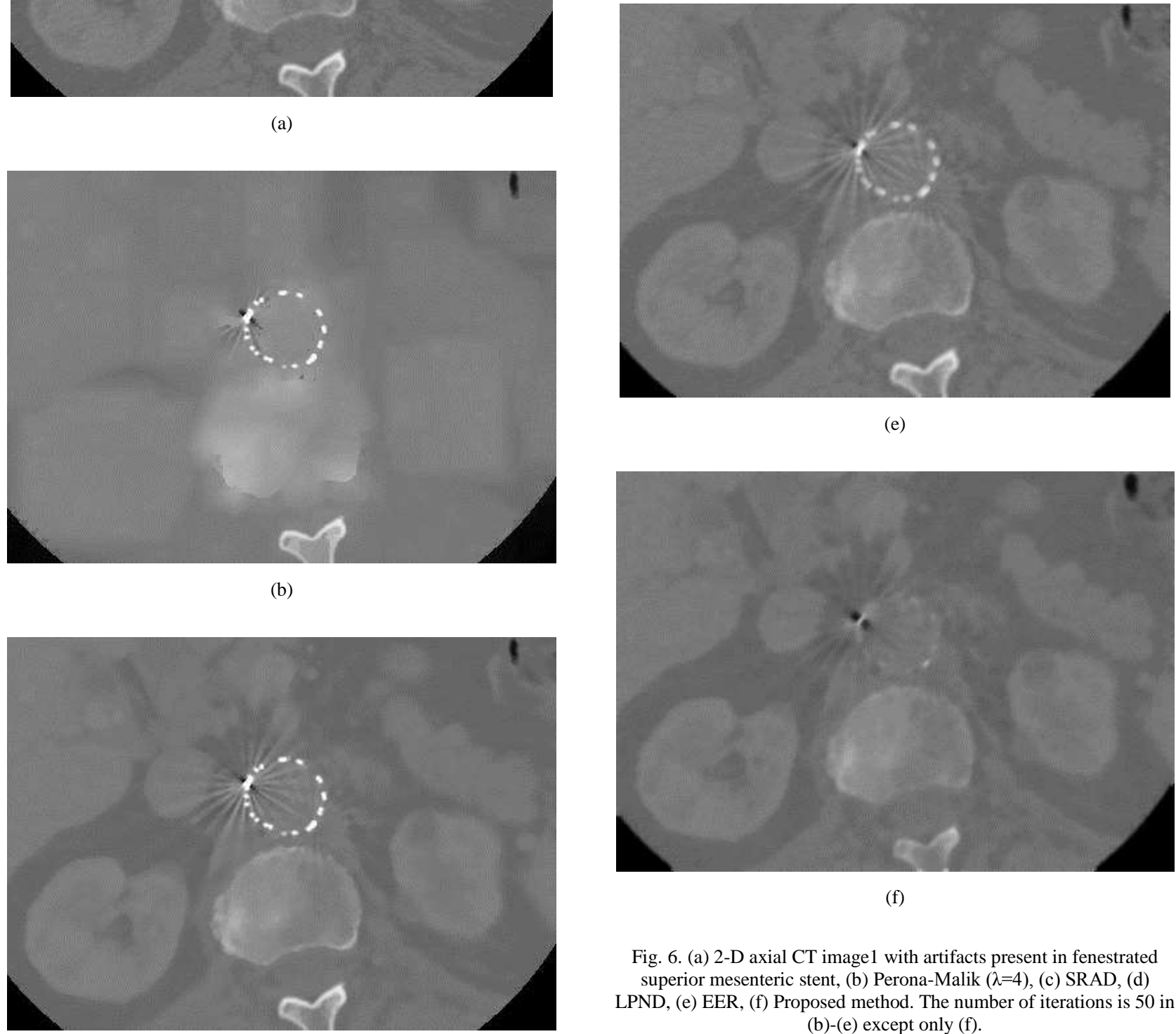

(e)

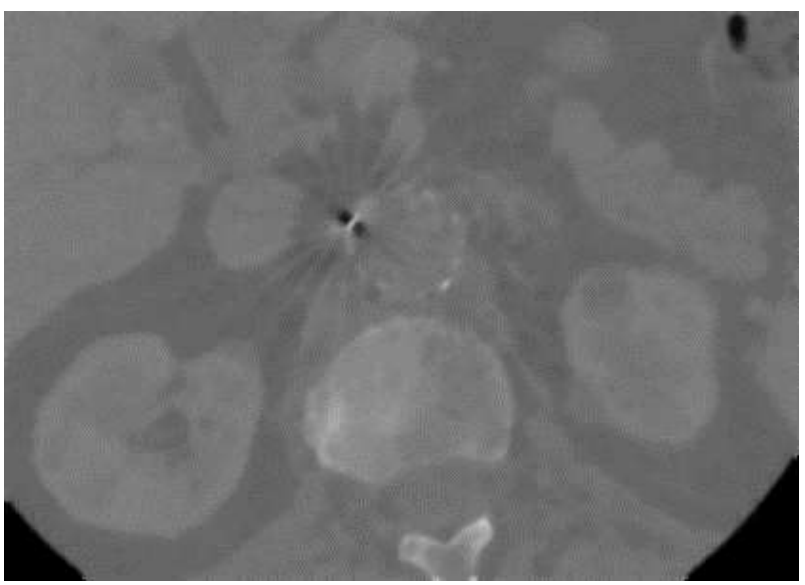

(f)

Fig. 6. (a) 2-D axial CT image1 with artifacts present in fenestrated superior mesenteric stent, (b) Perona-Malik ( $\lambda=4)$, (c) SRAD, (d) LPND, (e) EER, (f) Proposed method. The number of iterations is 50 in (b)-(e) except only (f).

(c) 
From figure 6 and 7, we can see that our proposed filtered image is much clearer than other existing filtered images that mean noise reduction capability is very high of the proposed method in homogeneous and edge portions. Existing filters are unable to reduce excessive edge noises and in the mean time those filters blur the image and lose the clinical information only except our investigated method.

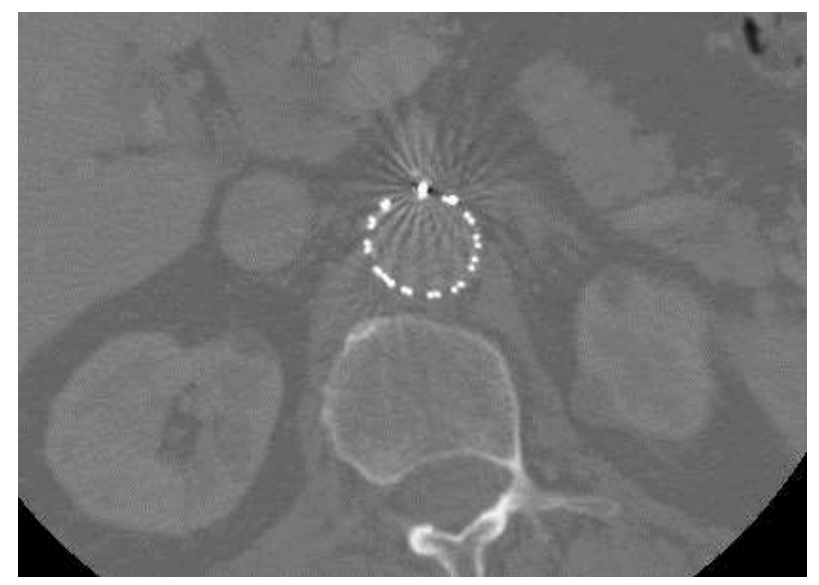

(a)

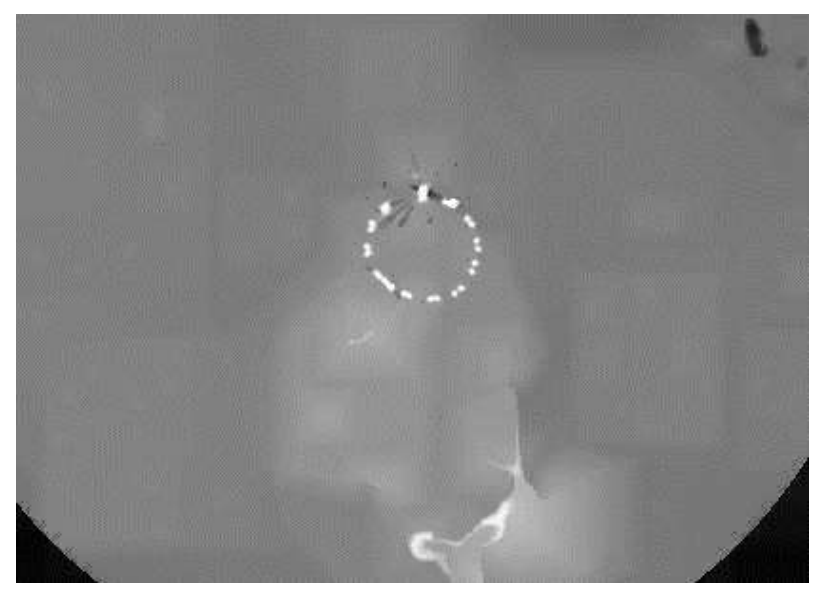

(b)

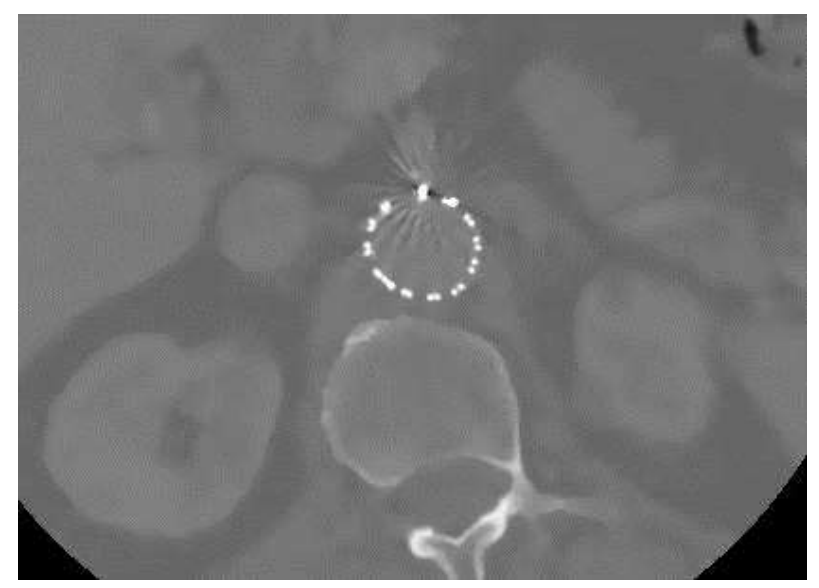

(c)

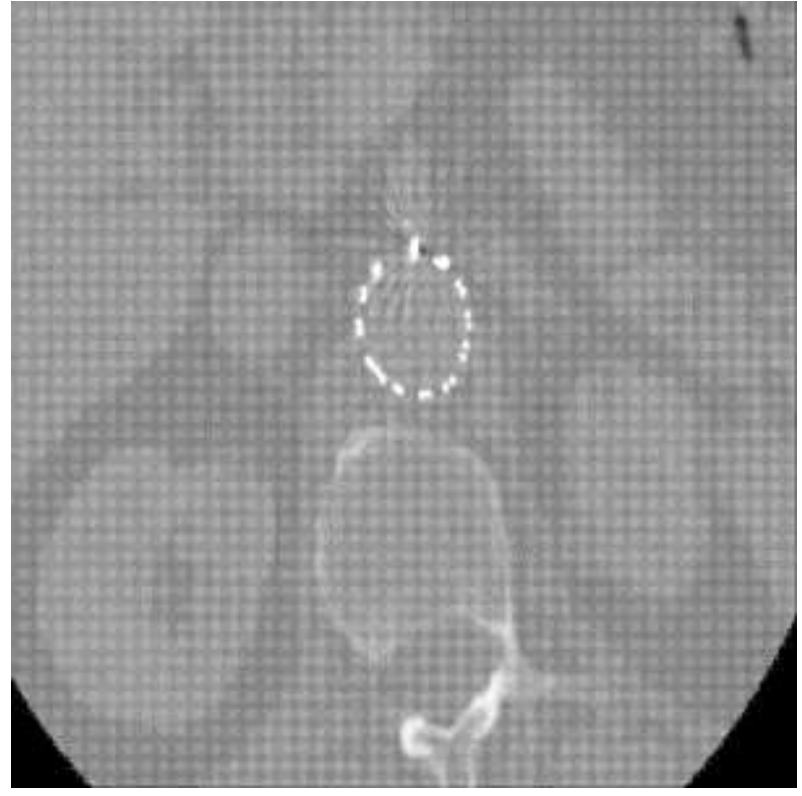

(d)

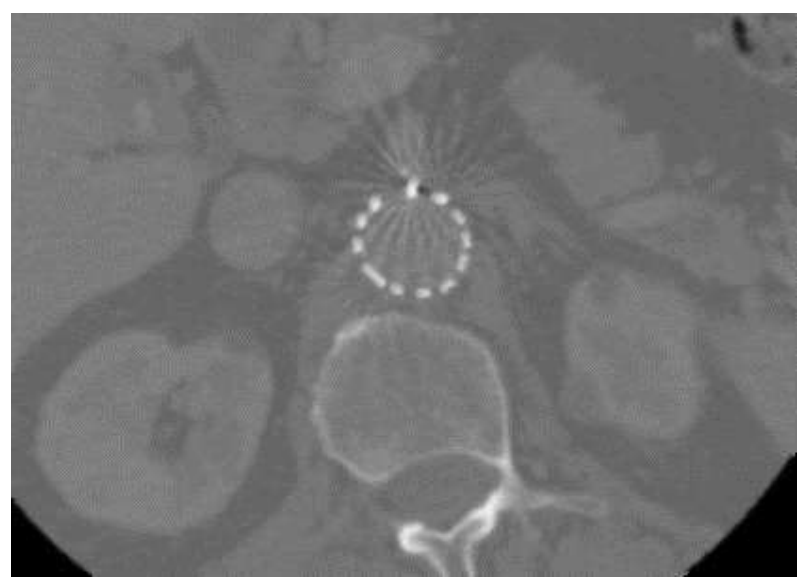

(e)

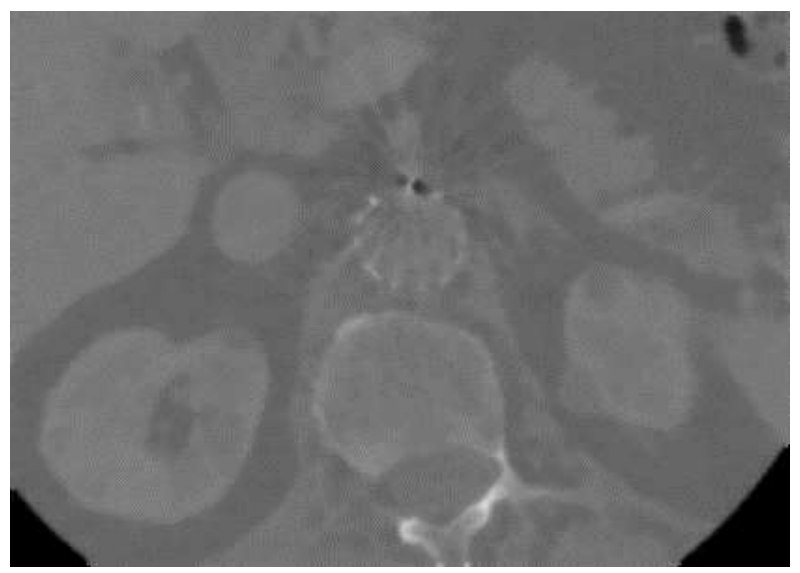

(f)

Fig. 7. (a) 2-D axial CT image2 with artifacts present in fenestrated superior mesenteric stent, (b) Perona-Malik $(\lambda=4)$, (c) SRAD, (d) LPND, (e) EER, (f) Proposed method. The number of iterations is 100 in (b)-(e) except only (f). 


\section{CONCLUSION}

In this paper, we proposed direction oriented iterative reconstruction filter with fuzzy logic which is depends on automated intensity of $\mathrm{CT}$ image for metal artifact reduction. The Experimental results on CT images show that the proposed method can reduce artifact effectively and significantly while edges, textures and point targets are smoothed as well as preserved. Simulated artifact corrupted CT images show better visual quality. This method mainly depends on the signal direction according to the magnitude variation in the CT images. Our proposed method is especially effective for highly inhomogeneous image and can be used widely for artifact and the calcification plaque strongly reduction and the enhancement of the structure of artifact affected CT images.

\section{ACKNOWLEDGEMENT}

We would like to thanks professor Dr. Zhonghua Sun for providing these images.

\section{REFERENCES}

[1] G N Hounsfield, "Computerized transverse axial scanning (tomography): Part 1. description of system", British Journal of Radiology, (1973, December), 46:1016-1022.

[2] Fessler J A, (2000), in J.MFritzpatrick andMSonka, eds, "Handbook of Medical Imaging", Vol. 2 SPIE Press Bellingham, WA chapter 1, pp. 1-70.

[3] LaRivière P J and Vargas P, M. J Flynn and J Hsieh, 'Medical Imaging 2006: Physics of Medical Imaging', 2006, Vol. 6142 Proc. SPIE pp. 61420X-1-61420X8.

[4] Oehler M and Buzug T M , Meth. Inform. Med. 2007, 46, 261-269.

[5] Müller J and Buzug T M, in E Samei and J Hsieh, eds, 'Medical Imaging 2009: Physics of Medical Imaging' 2009, Vol. 7258 Proc. SPIE pp. 72581Y-1-8.

[6] Bhateja, V., Devi, S.: An Improved Non-Linear Transformation Function for Enhancement of Mammographic Breast Masses. In: Proc. of (IEEE) 3rd International Conference on Electronics \& Computer Technology (ICECT 2011), Kanyakumari (India), 2011, vol. 5, pp. 341-346.

[7] Chen, L., Lu, G., Zhang, D.: Effects of Different Gabor Filter Parameters on Image Retrieval by Texture. In: Proc. of IEEE 10th International Conference on Multi-Media Modelling, Brisbane, Australia, (January 2004). pp. 273278.

[8] Perreault, S., Hébert, P.: Median Filtering in Constant Time. IEEE Trans. on Image Processing, 2007, 16(6), 2389-2394.

[9] Solbo, S., Eltoft, T.: Homomorphic Wavelet Based Statistical Despeckling of SAR Images. IEEE Trans. on Geoscience and Remote Sensing, 2004, 42(4), 711-721.

[10] Strela, V.: Denoising via Block Wiener Filtering in Wavelet Domain. In: 3rd European Congress of Mathematics, (July 2000), Barcelona, Spain.

[11] Kaur, A., Singh, K.: Speckle Noise Reduction by Using Wavelets. In: National Conference on Computational Instrumentation, Chandigarh, India, (March 2010), pp. 198-203.
[12] Mohideen, S.K., Perumal, S.A., Sathik, M.M.: Image Denoising Using Discrete Wavelet Transform. International Journal of Computer Science and Network Security, 2008, 8(1), 213-216.

[13] Solbo, S., Eltoft, T.: A Stationary Wavelet Domain Wiener Filter for Correlated Speckle. IEEE Trans. on Geoscience and Remote Sensing, 2008, 46(4), 1219-1230.

[14] Tong, Z., Chen, X., Akram, M.N., Aksnes, A.: Compound Speckle Characterization Method and Reduction by Optical Design. Journal of Display Technology, 2012, 8(3), 132-137.

[15] Jain, A., Bhateja, V.: A Novel Image Denoising Algorithm for Suppressing Mixture of Speckle and Impulse Noise in Spatial Domain. In: Proc. of IEEE 3rd International Conference on Electronics and Computer Technology, Kanyakumari, India, (April 2013), pp. 207-211.

[16] Singh, S., Jain, A., Bhateja, V.: A Comparative Evaluation of Various Despeckling Algorithms for Medical Images. In: Proc. of (ACMICPS) CUBE International Information Technology Conference \& Exhibition, Pune, India, (September 2012), pp. 32-37.

[17] Jain, A., Singh, S., Bhateja, V.: A Robust Approach for Denoising and Enhancement of Mammographic Breast Masses. International Journal on Convergence Computing, 2013, 1(1), 38-49.

[18] Bhateja, V., Urooj, S., Pandey, A., Misra, M., LayEkuakille, A.: A Polynomial Filtering Model for Enhancement of Mammogram Lesions. In: Proc. of IEEE International Symposium on Medical Measurements and Applications, Gatineau (Quebec), Canada, (May 2013), pp. 97-100.

[19] Bhateja, V., Urooj, S., Pandey, A., Misra, M., LayEkuakille, A.: Improvement of Masses Detection in Digital Mammograms employing Non-Linear Filtering. In: Proc. of IEEE International Multi-Conference on Automation, Computing, Control, Communication and Compressed Sensing, Palai-Kottayam, Kerala, India, (2013), pp. 406-408.

[20] Rahman, M. M., PK, M. K., Borucki, B., Nowinski, K.S.: Speckle noise reduction of ultrasound images using ExtraEnergy Reduction function, IEEE $2^{\text {nd }}$ International Conference on Informatics, Electronics and Vision, Dhaka, Bangladesh, (May 2013), pp. 1-6.

[21] Gupta, A., Tripathi, A., Bhateja, V.: Despeckling of SAR Images via an Improved Anisotropic Diffusion Algorithm. In: Satapathy, S.C., Udgata, S.K., Biswal, B.N. (eds.) Proceedings of Int. Conf. on Front. of Intell. Comput. AISC, Springer, Heidelberg (2013), vol. 199, pp. 747-754.

[22] Gupta, A., Ganguly, A., Bhateja, V.: A Noise Robust Edge Detector for Color Images using Hilbert Transform. In: Proc. of (IEEE) 3rd International Advance Computing Conference (IACC 2013), Ghaziabad (U.P.), India, 2013, pp. 1207-1212.

[23] Zhang, J., Hu, J.: Image Segmentation Based on 2D Otsu Method with Histogram Analysis. In: International Conference on Computer Science and Software Engineering, Wuhan, Hubei, (December 2008), pp. 105108.

[24] Sezgin, M., Sankur, B.: Survey Over Image Thresholding Techniques and Quantitative Performance Evaluation. Journal of Electronic Imaging, 2004, 13(1), 146-165.

[25] Azarbad, M., Ebrahimzade, M.A., Izadian, V.: Segmentation of Infrared Images and Objectives Detection Using Maximum Entropy Method Based on the Bee Algorithm. International Journal of Computer Information Systems and Industrial Management Applications, 2011, 3 , 26-33. 
[26] Gupta, A., Ganguly, A., Bhateja, V.: An Edge Detection Approach for Images Contaminated with Gaussian and Impulse Noises. In: Mohan, S., Suresh Kumar, S. (eds.) ICSIP 2012. LNEE, Springer, Heidelberg (2012), vol. 222, pp. 523-533.

[27] Jain, A., Bhateja, V.: A Versatile Denoising Method for Images Contaminated with Gaussian Noise. In: Proc. of CUBE Int. Information Technology Conf. \& Exhibition, Pune, India, 2012, pp. 65-68.

[28] Chumning, H., Huadong., C.W.: Edge preservation evaluation of digital speckle filters. In: IEEE International Geoscience and Remote Sensing Symposium, June 24-28, 2002, vol. 4, pp. 2471-2473.

[29] Rahman, M. M., PK, M. K., Aziz, A., Arefin M.G. and Uddin, M. S., "Adaptive anisotropic diffusion filter for speckle noise reduction for ultrasound images", Inderscience Publishers International Journal on Convergence Computing, 2013, 1(1), 50-59.

[30] Jain, A., Bhateja, V.: A Full-Reference Image Quality Metric for Objective Evaluation in Spatial Domain. In: Proc. of IEEE International Conference on Communication and Industrial Application (ICCIA), Kolkata (W. B.), India, 2011, pp. 91-95.

[31] Guo, Y., Cheng, H.D., Tian, J., Zhang, Y.: A Novel Approach to Speckle Reduction to Ultrasound Image. In: Proc. of 11th Joint Conference on Information Sciences, China, (December 2008), pp. 1-6.

[32] Bhateja, V., Misra, M., Urooj, S., Lay-Ekuakille, A.: A Robust Polynomial Filtering Framework for Mammographic Image Enhancement from Biomedical Sensors. IEEE Sensors Journal, 2013, 1-10.

[33] Trujillo, C. and Garcia-Sucerquia, J.Accelerated Numerical Processing of Electronically Recorded Holograms With Reduced Speckle Noise", IEEE Trans. On Image Processing, 2013, 22(9), pp. 3528 - 3537.

[34] P. Perona and J. Malik., "Scale space and edge detection using anisotropic diffusion," IEEE Trans on pattern analysis and machine, 1990, vol. 12, no.7, pp. 629-639.

[35] F. Zhang, Y. M. Yoo, L. M. Koh and Y. Kim, "Nonlinear Diffusion in Laplacian Pyramid Domain for Ultrasonic Speckle Reduction," IEEE Trans. On Medical Imaging, 2007, vol. 26, no. 2, Feb 2007.

[36] M. J. Black, G. Sapiro, D. H. Marimont and D. Heeger, "Robust anisotropic diffusion," IEEE Trans. Image Process., Mar. 1998, vol. 7, no. 3, pp. 421-432,.

[37] Y. J. Yu and S. T. Acton, "Speckle reducing anisotropic diffusion,” IEEE Trans. Image Process, Nov. 2002, vol. 11 no. 11, pp. 1260-1270.

[38] Wang, Y., "The real-time process algebra (RTPA)". Annals of Software Engineering: An International Journal, (2002, October), 14, 235-274. Kluwer Academic Publishers.

\section{Authors' Profiles}

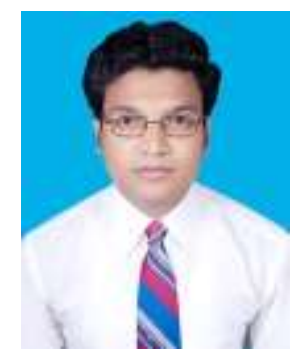

Mithun Kumar PK was born in Rajshahi, Bangladesh in 1989. He received the B.Sc Engineering degree in Computer Science \& Engineering from Mawlana Bhashani Science and Technology University, Tangail, Dhaka, Bangladesh. His main areas of research interests are image analysis, image processing \& medical image processing, pattern recognition, 3D visualization, Segmentation, Filter Optimization. He has many international journal and conference publications and he is a regular reviewer of IET Image Processing journal, International Arab Journal of Information Technology, Journal of Media and Communication Studies. He is also a Technical Committee member at International Arab Journal of Information Technology.

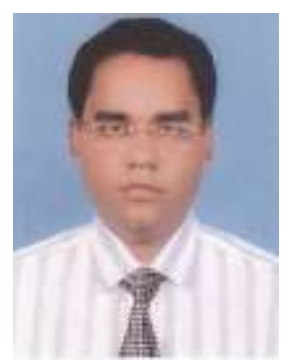

Mohammad Motiur Rahman is an associate professor of Department of Computer Science \& Engineering in Mawlana Bhashani Science and Technology University, Bangladesh. He received the B.Sc Engg. \& M.S degree in Computer Science \& Engineering from Jahangirnagar University, Dhaka, Bangladesh, in $1995 \& 2001$, Where he is currently pursuing the Ph.D. degree. His research interests include digital image processing, medical image processing, computer vision $\&$ digital electronics. He has many international journal and conference publications in renowned journals.

How to cite this paper: Mithun Kumar PK, Mohammad Motiur Rahman,"Metal Artifact Reduction from Computed Tomography (CT) Images using Directional Restoration Filter", IJITCS, vol.6, no.6, pp.47-54, 2014. DOI: $10.5815 /$ ijitcs.2014.06.07 